\title{
An investigative study in Dima Hasao District, Assam: Study of association of alcoholic drink and ALD using three biomarkers of liver-AST, ALT and Bilirubin.
}

\author{
Mandira Lohar ${ }^{1}$, Pimily Langthasa ${ }^{2}$ \\ Molecular and Cell Biology Lab., Deptt. of Life Science and Bioinformatics Assam University Silchar.
}

\begin{abstract}
Liver is one of the vital organ of the body and any kind of chemical exposure to it may lead to its toxicity. The use of alcohol is being a common practice around the world alternating the life style of the people from normal to dependency towards it. As a result, people are emerging with alcoholic liver health issues. Many liver biomarkers like AST, ALT, GGT, Bilirubin etc. are being used to assess the liver health status but biopsy is the most accurate one. Till date ALT, AST and Bilirubin is considered as the primary LFT test to evaluate any kind of liver injury. The people of Dima Hasao District, Assam, India practices a typical traditional alcoholic drink called Judima and Juharu which they consider it to be harmless to liver health. Therefore, the main objective of this study is to assess the liver health status of the people of Dima Hasao District, using three circulating biomarkers of liver- AST, ALT and Bilirubin. A group of 35 people are involved in the present study categorized as daily alcohol users $(n=19)$ and $(n=15)$ as non-alcohol users. The result are shown in mean $\pm S E$. The data are analyzed in Statistical Analysis Software and the result is interpreted by Student t-test. The result shows significant increase of ALT with $p<0.01$, AST with $p<0.001$ and Bilirubin with $p<0.05$ in daily alcohol users compared to non-alcohol users. Thus, elevation of liver enzymes in blood serum indicates acute liver injury amongst the people of this district drinking Judima and Juharu alcoholic drink and they should undergo medications to arrest the serious hepatic toxicity problem caused by this alcoholic drink.
\end{abstract}

Key words: Alcoholic Liver Disease, Hepatic Damage, ALT, AST and Bilirubin.

\section{Introduction:}

With the increasing life style factors in recent years, a large number of people around the world have been emerging with alcoholic liver diseases (ALD) like alcoholic fatty liver, alcoholic hepatitis and alcoholic liver cirrhosis (Dasarathy et al., 2010). Liver is one of the most vital organ of the body and any kind of harmful chemical exposure may lead to hepatic toxicity. Alcoholic liver disease (ALD) represents a spectrum of clinical illness and morphological changes that range from fatty liver to hepatic inflammation and necrosis (alcoholic hepatitis) to progressive fibrosis (alcoholic cirrhosis). Lieber, in the year 2003 also reported the association of alcohol and liver disease. In recent years, several biochemical tests such as AST, ALT, GGT, Bilirubin, Albumin etc (Hyder et al., 2013) have been developed to evaluate and manage the patients hepatic dysfunction. Marked level of aminotransferases in the appropriate clinical context indicate acute cell necrosis caused by viral infection, drugs toxins, alcohol or ischemia (Desmond Burke,2002). Liver biopsy is the most accurate way to assess liver diseases (Bird, 1993). But due to its invasive nature, such screening process to assess liver injury is practically inconvenient. Effects of alcohol consumption and alcoholic liver diseases with non-invasive markers have been found in multiple studies (Savoleinan V, Perola M, Lalu K et al., 1995).

The district of Dima Hasao, Assam, India is a land of different tribal communities where alcohol consumption is a common practice and hence alcohol related mortality and morbidity has become a major problem of this district. Liver being one of the most vital organ, any kind of damages to it may lead to serious negative health consequences. Therefore, to evaluate the problem of liver related diseases caused by this typical alcoholic drink (Judima and Juharu with an alcoholic content of 18\% and 25\%), it has become very much essential to study liver health status of the people of this district. With this objective in mind, the present investigative study has been carried out in Dima Hasao District of Assam. India.

\section{Materials And Methods:}

A group of 35 male daily alcohol user and non-alcoholics was included in the present study. Among the alcoholics $(\mathrm{n}=19)$, people those who has been drinking for the past 8-15years, aged between 25-50 years were followed to assess liver injury status comparing with non-alcoholics $(n=15)$. A 5-minutes interview was taken from each subjects through a prepared standard questionnaire which included demographic characteristics, habitual information, health associated complaints and clinical test report. To assess the liver injury status Liver Function Test (LFT) was conducted in Haflong Civil Hospital, Haflong by using three biomarkers - AST 
(Aspartate Amino Transaninase), ALT (Alanine Amino Transaminase) following Reitman and Frankel method (1957) and Bilirubin test was conducted following Jendrassik and Groff,s method (1938) .

\section{Quantitative Analysis:}

Quantitative data are expressed as mean \pm SE and analyzed by Student t-test (Turkey's multiple comparison test for pair wise comparison between study groups). Differences were considered statistically significant when $\mathrm{p}<0.05$. Statistical analysis was performed using Graph Pad Prism version 4.00 software, San Diego California USA.

\section{Result:}

The present study involves a group of 35 people with $(n=19)$ as daily alcohol users and $(n=15)$ as nonalcoholics. Table 1.1 shows the patients characteristics with or without the habit of alcohol consumption. Serum transaminases [Aspartate Amino Transaminase (AST), Alanine Amino Transaminase (ALT)] and Bilirubin were found to be significantly higher in the alcoholic group when compared with the non-alcoholic group reflecting alcohol abuse and associated liver diseases as shown in Table 1.2. Association of Increased level of AST, ALT and Bilirubin amongst the alcoholics with their habit of drinking pattern is shown in Table1.3. Therefore, the present study reveals that the alcoholics are suffering from acute liver injuries drinking this typical alcoholic drink called Judima and Juharu and should undergo immediate medications and nutrients supplements for early recovery from hepatic toxicity. (Lieber, 2003).

V. Figures And Tables

Table 1.1: Patient Characteristics

\begin{tabular}{|l|l|l|}
\hline & Alcoholics $(\mathrm{n}=19)$ & Non-alcoholics $(\mathrm{n}=15)$ \\
\hline Atudied Characteristics & $37.16 \pm 1.07$ & $37.16 \pm 1.97$ \\
\hline & & \\
\hline Body Mass Index $\left(\mathrm{kg} / \mathrm{m}^{2}\right)$ & $21.11 \pm 0.57$ & $23 \pm 0.76$ \\
\hline Alcohol Intake (ml/day) & & \\
\hline & $539.47 \pm 87.64$ & $225 \pm 33.35$ \\
\hline Years of Alcohol Consumption & $15.58 \pm 1.63$ & \\
\hline & & $3.17 \pm 0.36$ \\
\hline Haemoglobin $(\mathrm{gm} / \mathrm{dl})$ & $10.79 \pm 0.30$ & \\
\hline
\end{tabular}

Datas are in Mean \pm SE

Table 1.2: Assessment of Liver Injury through three circulating biomarkers of Liver.

\begin{tabular}{|l|l|l|}
\hline & Alcoholics $(\mathrm{n}=19)$ & Non-alcoholics $(\mathrm{n}=15)$ \\
\hline Biomarkers & $94.4 \pm 12.4^{* * *}$ & $40.27 \pm 6.47$ \\
\hline & & \\
\hline ALT (U/l) & $61.9 \pm 9.45^{* *}$ & $30.2 \pm 5.56$ \\
\hline & & \\
\hline Bilirubin $(\mathrm{mg} / \mathrm{dl})$ & $2.8 \pm 0.6^{*}$ & $1.33 \pm 0.23$ \\
\hline
\end{tabular}

$\mathrm{P}<0.05=*, \mathrm{p}<0.01=^{* *}, \mathrm{p}<0.001=* * *$

Table 1.3: Association of Liver Injury with Drinking Pattern indicated by three Circulating Biomarkers

\begin{tabular}{|l|l|l|l|}
\hline Alcohol Consumption (ml/day) & AST (U/I) & ALT (U/l) & Bilirubin (mg/dl) \\
\hline $125-375$ & $97.77 \pm 53.58$ & $62.73 \pm 42.62$ & $2.87 \pm 3.035$ \\
\hline $750-1125$ & $108.83 \pm 53.25$ & $78.83 \pm 41.71$ & $2.91 \pm 2.15$ \\
\hline
\end{tabular}

Figure1.1: Graphs showing the effect of liver injury using three biomarkers - AST, ALT and Bilirubin.

AST

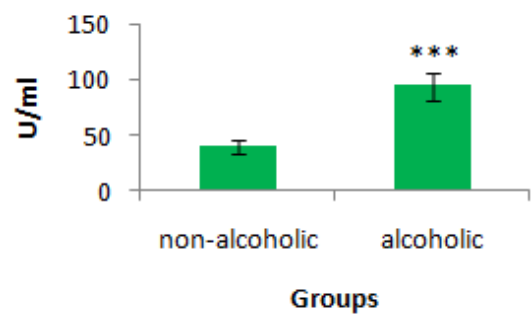

ALT

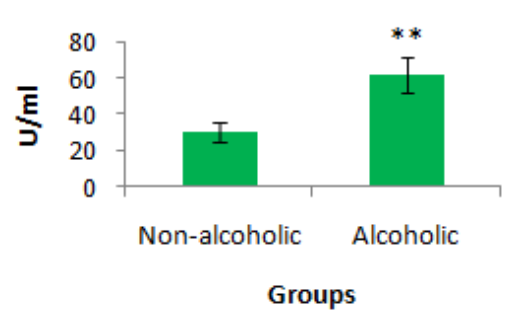




\section{Bilirubin}

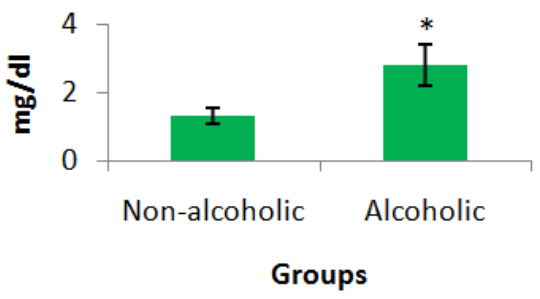

\section{Discussion}

Alcoholic liver disease (ALD) represents a spectrum of clinical and morphological changes that range from fatty liver to hepatic inflammation and necrosis (alcoholic hepatitis) to progressive fibrosis (alcoholic cirrhosis) (Gramenzi et al, 2006). Studies reveals that only a minority of alcoholics eventually develops major complications such as liver cirrhosis (Lieber, 1992) and there is an immediate need of arresting or reversing such kind of serious organ damage at an early stage. Individual differences in the rate of ethanol metabolism and the severity of alcohol induced liver damage are, in a part genetically determined, but no such genetic markers are available to assess that level (Lumeng and Crabb, 1994).

At present, there are several types of biochemical tests available using blood serum which can measure the degree of liver ailments. Some of the biochemical tests are AST, ALT, GGT, Albumin, Bilirubin, Prothrombin, hepatitis etc. (Reitman et al, 1957 and Jendrasikk et al., 1938) which serves the purpose of preliminary detection of the progression of liver damage to cirrhosis stage. Although Liver Biopsy is the most accurate one but due to its invasive nature it is not a suggestive method. The present study involves using the three circulating biomarkers of blood serum i.e. AST, ALT and Bilirubin to assess the degree of liver damage. It was reported by Hyder et al., 2013 that high rise of AST and ALT indicates viral hepatitis, alcoholic liver diseases (ALD) and cirrhosis. In the present study it is observed that there was a high elevation of AST and ALT in the blood serum among the alcoholics compared to non-alcoholics indicating the chronic effect of alcohol upon the liver health condition. The mode of action of alcohol on these markers is unknown and also the mechanism involved, but it should be borne in mind that in present study in assessing the significance of test result association of alcohol should be taken into account.

\section{Conclusion}

Present study reveals acute hepatic toxicity amongst the study groups practicing alcohol (Judima and Juharu) consumption compared to non-alcohol consumers which directly indicates the potential of inducing hepatic toxicity by this typical traditional alcoholic drink. Therefore, people of this tribal community which they consider it to be harmless should be taken care of. They should undergo medications to overcome their liver ailments.

\section{Acknowledgement}

The authors are thankful to the Unit of Haflong Civil Hospital, Haflong and the volunteers for the current study.

\section{Referrences}

[1]. O'Shea RS, Dasarathy S, McCullough AJ, 2010. "Alcoholic liver disease" (PDF). Hepatology 51 (1): 307-28.

[2]. Hyder M A, Hasan M, Mohieldein A, Eur. Jr. of Expt. Bio. 2013, 3(2): 280-284

[3]. Desmond Burke M, Clin Lab Med , 2002:22: 377-39

[4]. Bird GL. Investigation of alcoholic liver disease: Baillier Clin Gastroenterol 1993;7:663-82

[5]. Savolainen, E.-R., Goldberg, B., Leo, M. A., Velez, M. and Lieber, C. S. (1984).Diagnostic value of serum procollagen peptide measurements in alcoholic liver disease. Alcoholism: Clinical and Experimental Research 8,384-389.

[6]. Reitman, S. and S. Frankel:.American. Journal of Clinical Pathology. 1957, 28: 56-62.

[7]. Jendrassik, L; Grof,P (1938): Bilirubin Kit, Biochem 2,297:81.

[8]. Lieber, C.S. (2003): Relationships between nutrition, alcohol use, and liver disease. Alcohol Research \& Health 27(3):220-231.

[9]. Lieber, C. S. (1992):Alcohol and the liver. In Medical and Nutritional Complications of Alcoholism: Mechanism and Management.

[10]. Gremenzi A., Caputo F, Biselli M Aliment: Pharmacol Ther 2006,24,:1151-1161.

[11]. Lumeng, L. and Crabb, D. W. (1994: Genetic aspects and risk factors in alcoholism and alcoholic liver disease. Gastroenterology 107, 572-578. 